\title{
Probing the Stellar Surface of HD209458 from Multicolor Transit Observations
}

\author{
H.J. Deeg ${ }^{\mathrm{a}, \mathrm{b}, 1}$ R. Garrido ${ }^{\mathrm{a}}$ A. Claret $^{\mathrm{a}}$ \\ ${ }^{a}$ Instituto de Astrofísica de Andalucía, C. Bajo de Huétor 24, Granada, Spain \\ b Instituto de Astrofísica de Canarias, C. Vía Láctea, La Laguna, Tenerife, Spain
}

\begin{abstract}
Multicolor photometric observations of a planetary transit in the system HD 209458 are analyzed. The observations, made in the Strömgren photometric system, allowed a recalculation of the basic physical properties of the star-planet system. This includes derivation of linear limb-darkening values of HD 209458, which is the first time that a limb-darkening sequence has observationally been determined for a star other than the Sun. As the derived physical properties depend on assumptions that are currently known with limited precision only, scaling relations between derived parameters and assumptions are given. The observed limb-darkening is in good agreement with theoretical predictions from evolutionary stellar models combined with ATLAS model atmospheres, verifying these models for the temperature $\left(T_{\text {eff }} \approx 6000 K\right)$, surface gravity $(\log g \approx 4.3)$ and mass $\left(\approx 1.2 M_{\odot}\right)$ of HD 209458 .
\end{abstract}

Key words: planetary systems, stars: atmospheres, stars: fundamental parameters, stars: individual (HD 209458), techniques: photometric

PACS: 95.75.-z, 97.10.-q, 97.10.Ex, 97.10.Pg, 97.20.Jg, 97.82.+k

\section{Introduction}

The discovery of the first extrasolar planet transiting its central star, HD 209458 (Charbonneau et al., 2000; Henry et al., 2000) opened a new way to study these objects through the analysis of the light curve that is generated during a transit. Similarly, details about the central star may be revealed. Here we present observations of a transit of HD 209458 that were taken simultaneously in the four Strömgren colors. The primary motivation was a study of

$\overline{1}$ E-mail: users hdeeg, garrido, claret at user@iaa.es 
the specific intensity distribution of the stellar surface of HD 209458 for comparison with existing models of stellar atmospheres through the derivation of limb-darkening coefficients. A transit light curve provides in its central part between second and third contact - a direct measure of the specific intensity behind the planet. The specific intensity behind the ingress and egress zones is also accessible, through models of transit light curves. A planetary transit profile gives significant advantages against efforts to derive intensities across the stellar disc from light curves of eclipsing binaries: in transits, a small dark disk passes in front of the star, whereas eclipsing binaries cause ambiguities from the presence of two luminous sources, frequently of similar size range, and often aggravated by light reflection between the two components, or by gravitational darkening in the case of fast rotators.

The major difference between the data presented here and the $B V R I Z$ multicolor transit observations by Jha et al. (2000) is the different wavelength span, with our data taken in the Strömgren photometric system. Also, our data were taken in all colors simultaneously, whereas Jha et al. used a CCD camera cycling sequentially through the $V, R$ and $I$ filters, with data in further colors obtained at telescopes in different locations. In the following analysis we then show, that transit light curves do not only allow the derivation of parameters of the extrasolar planet, but impose severe constraints on the mass-to-radius relationship of the central star as well. Furthermore, derived parameters of the star-planet system are presented in a way that is independent of assumptions based on approximately known values, such as the stellar mass. Finally, linear limb-darkening coefficients are given as a function of the stellar radius and mass of HD 209458 and compared to stellar and atmosphere models. This is the first time that a color sequence of limb-darkening coefficients for a single star besides the Sun has been derived from observations.

\section{Data acquisition}

The observations of HD 209458 reported here were performed in the night of 25-26 July 2000 with the $0.9 \mathrm{~m}$ telescope of the Observatorio Sierra Nevada, located near Granada, Spain at an altitude of $2900 \mathrm{~m}$. The telescope is equipped with a 4 channel photometer, allowing simultaneous observations in all colors of the $u, v, b, y$ Strömgren photometric system. This instrument is similar to that of the Strömgren Automatic Telescope at La Silla (Gronbech et al., 1976). The observing cycle consisted of a $30 \mathrm{sec}$ sky-exposure, $20 \mathrm{sec}$ exposures of standard stars SAO 107675 and SAO 107561, and a 40 sec exposure of the object, HD 209458. During the ingress and egress of the transit, for about 40

minutes each time, only HD 209458 was observed with repeated 40 second exposures. During the flat bottom part of the transit, the second comparison star was left out. These modifications served to increase the time resolution, 


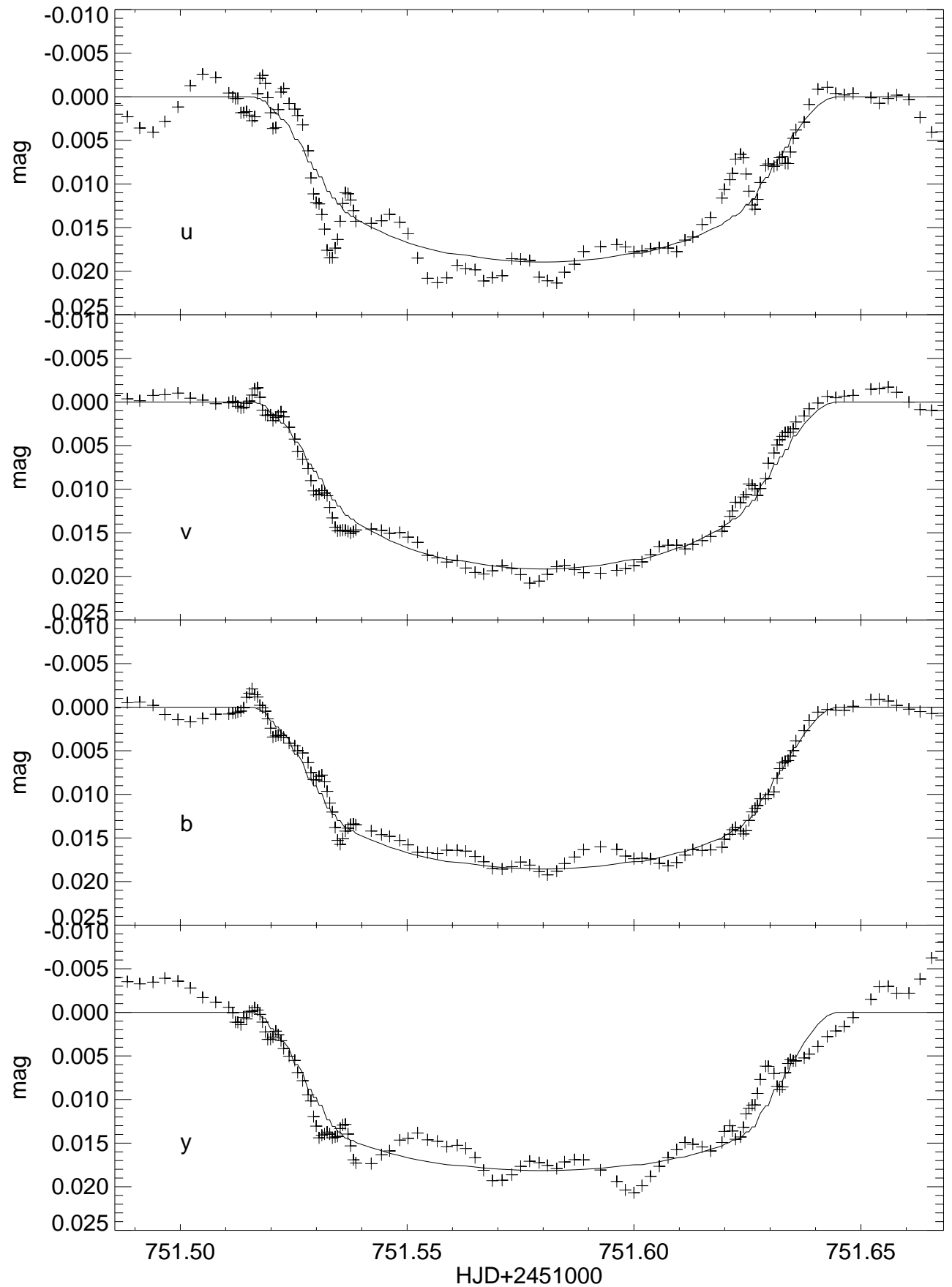

Fig. 1. $u, v, b$, and $y$ light curves that were used as the basis for the analysis (crosses). They are smoothed by a triangular kernel with a width of 5 data points. The solid lines are model light curves for a star-planet system with the parameters given in Table 3 . 
and were justifiable since the night was photometric, with last quarter moon, and the mid-transit occurred within a few minutes of the meridian crossing of HD 209458, leading to equal airmasses at ingress and egress. Data reduction was performed at the telescope Macintosh control computer, with the derivation of an extinction function based on the comparison stars. Differential photometry was then performed with respect to the first comparison star. Resulting lightcurves are available in electronic Table 1. Magnitude differences between both comparison stars indicate a photometric precision of about 4 mmag for the $u$ and $y$ bands and 2 mmag for the $v$ and $b$ ones per single measurement, with the better results in the two latter bands simply being due to higher photon count rates. After removal of poorer data at the begin of the observations, the transit light curves used in the analysis contain 129 data points in all colors, with 29 off-transit points, which were used to set the magnitude zero-point ${ }^{2}$. In the off-transit part, the rms noise is: $4.2 \mathrm{mmag}$ for $u, 2.2 \mathrm{mmag}$ for $v, 1.9 \mathrm{mmag}$ for $b$ and $3.4 \mathrm{mmag}$ for $y$. For the subsequent analysis, the light curves were smoothed with a triangular kernel of 5 elements width, obtained through two successive boxcar smoothings with a 3 element rectangular kernel. The off-transit rms of the resulting light curves, shown in Fig. 1, is then: $u$ : $1.8 \mathrm{mmag}, v$ : $0.72 \mathrm{mmag}, b$ : $0.89 \mathrm{mmag}, y: 2.5 \mathrm{mmag}$. Some parts of our analysis are based on a combined $v+b$ light curve, for which a lower rms of $0.65 \mathrm{mmag}$ was attained. Similar observations as reported here were performed in the night of July 18-19, 2000 as well, but increasingly hazy weather conditions and a nearly full moon significantly degraded these observations, leading us to concentrate on the data from 26 July.

\section{Analysis of the transit}

\subsection{Setup of model fits to the transit}

A convenient set of 'primary transit parameters', from which other frequently used parameters (like the planet's orbital inclination $i$ or the orbital half axis) can easily be derived, may be selected as follows:

$P$ : orbital period

$T_{c}$ : epoch of the planetary transit

$M_{*}$ : stellar mass

$R_{*}:$ stellar radius

$R_{\mathrm{pl}}$ : effective planetary radius, which is the radius of an opaque disk occulting

$\overline{2}$ For the $y$ filter, shorter term variations not well described by the extinction function required offsets from the off-transit zero-point found through adjustment of the best fitting planet size (see Fig. 6) to that of the other colors 
the same amount of light as the planet.

$b$ : latitude of the transit across the stellar disk in stellar surface coordinates $w$ : linear limb darkening coefficient.

$w$ is representative for some descriptor of the intensity distribution across the stellar disk. Here, we will use the common linear limb-darkening expression, defined through $I(\cos \theta)=I(0)[1-w(1-\cos \theta)]$, where $\theta$ is the angle between the stellar surface and the line of sight. The use of a linear limb-darkening expression for HD 209458 is justified, since it gives a good description of surface intensities for stars with effective temperatures between 5000 and $6000 \mathrm{~K}$, and $T_{\text {eff }}=6000 \mathrm{~K}$ for HD 209458 (Mazeh et al., 2000).

Among the primary parameters, only the orbital period and the epoch are known with high precision (Soderhjelm, 1999; Castellano et al., 2000; Robichon and Arenou, 2000). For this work, a period of 3.52474 days and an epoch of HJD 2451659.93675 from HST observations by Brown et al. (2001) was used, and - upon excellent agreement with our data - no attempt for a new derivation has been undertaken. Furthermore, estimates with a precision of about $10 \%$ exist for $R_{*}$ and $M_{*}$, with $R_{*}=1.2 R_{\odot}$ and $M_{*}=1.1 R_{\odot}$ (Mazeh et al., 2000, and references therein).

A boundary condition that allows a reduction in the number of free parameters is given by the total length of the transit, which is the time $T_{14}$ between the first and the last - fourth - contact of star and transiting planet. The observations by Brown et al. (2001) give a value for $T_{14}$ of 0.12795 days, which is in excellent agreement with our data. $T_{14}$ is given by the following expression, whose derivation is shown in Fig. 2:

$$
T_{14}=\frac{2 R_{*}}{v_{\mathrm{orb}}\left(M_{*}, P\right)}\left[\cos ^{2} b+\left(\frac{R_{\mathrm{pl}}}{R_{*}}\right)^{2}+\frac{2 R_{\mathrm{pl}}}{R_{*}}\right]^{1 / 2}
$$

where the planet's orbital velocity $v_{\text {orb }}$ depends in circular orbits ${ }^{3}$ on $M_{*}$ and $P$ as $v_{\text {orb }} \propto\left(M_{*} / P\right)^{1 / 3}$. With $T_{14}$ known, Eq.(1) can be solved for $\cos b$ as a function of $R_{*}, M_{*}$ and $R_{\mathrm{pl}}$ :

$$
\cos b=\left[\left(\frac{T_{14} v_{\mathrm{orb}}}{2 R_{*}}\right)^{2}-\left(\frac{R_{\mathrm{pl}}}{R_{*}}\right)^{2}-\frac{2 R_{\mathrm{pl}}}{R_{*}}\right]^{1 / 2}
$$

which corresponds to physically meaningful solutions only for $0 \leq \cos b \leq 1$.

$\overline{3}$ For the case of HD 209458b - a planet that is on a very close orbit around its central star - the actual $T_{14}$ will be, in the worst case, 1.0024 times longer than derived by Eq.(1) 


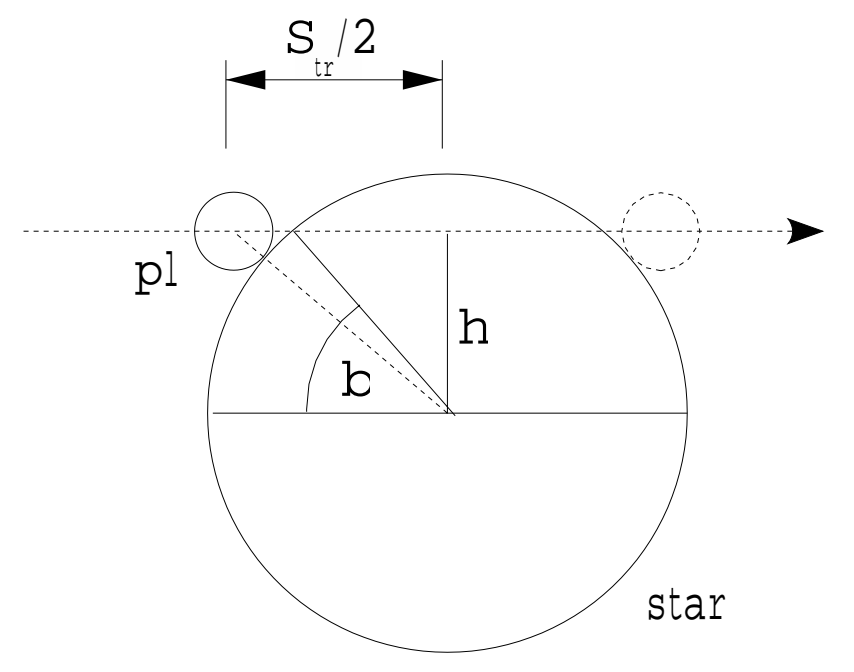

Fig. 2. The total transit duration $T_{14}=S_{\text {tr }} / v_{\text {orb }}$ depends on the lateral length of the transit $S_{\mathrm{tr}}$ as follows: $\left(S_{\mathrm{tr}} / 2\right)^{2}+h^{2}=\left(R_{\mathrm{pl}}+R_{*}\right)^{2}$, with $R_{\mathrm{pl}}$ and $R_{*}$ being the radii of the planet and of the star. If $h \leq R_{*}$, then $h$ may be expressed in terms of the latitude $b$ of the transit across the surface of the star: $h=R_{*} \sin b$, and consequently, $h^{2}=R_{*}^{2}\left(1-\cos ^{2} b\right)$. Solving this for $S_{\text {tr }}$ leads then to Eq.(1).

For a given combination of the parameters $\cos b, R_{*}, R_{\mathrm{pl}}$, an estimate for the limb-darkening coefficient $w$ can then be obtained as follows:

The relative brightness change $\Delta L / L$ at the center of a transit, assuming the linear limb-darkening expression, is given by:

$$
\Delta L / L=\left(\frac{R_{\mathrm{pl}}}{R_{*}}\right)^{2} \frac{3}{(3-w)}[1-w(1-\cos b)]
$$

which can be derived from the linear limb-darkening equation, with $\theta=b$ at the center of a transit. With an estimate of $\Delta L / L$ taken from the observed light curve, the above equation can be solved for $w$ :

$$
w=\frac{3\left[\left(R_{\mathrm{pl}} / R_{*}\right)^{2}-\Delta L / L\right]}{3\left(R_{\mathrm{pl}} / R_{*}\right)^{2}(1-\cos b)-\Delta L / L}
$$

Fits to the light curve were performed using the transit modeling code UTM, which calculates light curves following a set of input parameters, and the fitting routine 'UFIT' 4 , which wraps around UTM and provides UTM with

$\overline{4}$ UTM (Universal Transit Modeler) and UFIT (Universal Fitter) are written in IDL v.5 code and are available from HJD at http://www.iac.es/galeria/hdeeg/idl_hans_lib/utm/. UTM can be used in stand-alone mode to produce transit light curves, or within the fitting routine UFIT. UTM calculates the light curves of transits from pixelized representations of projections of the stellar surface and the transiting object. For both UFIT and UTM, setup files are used to completely describe a transit configuration, and allow 


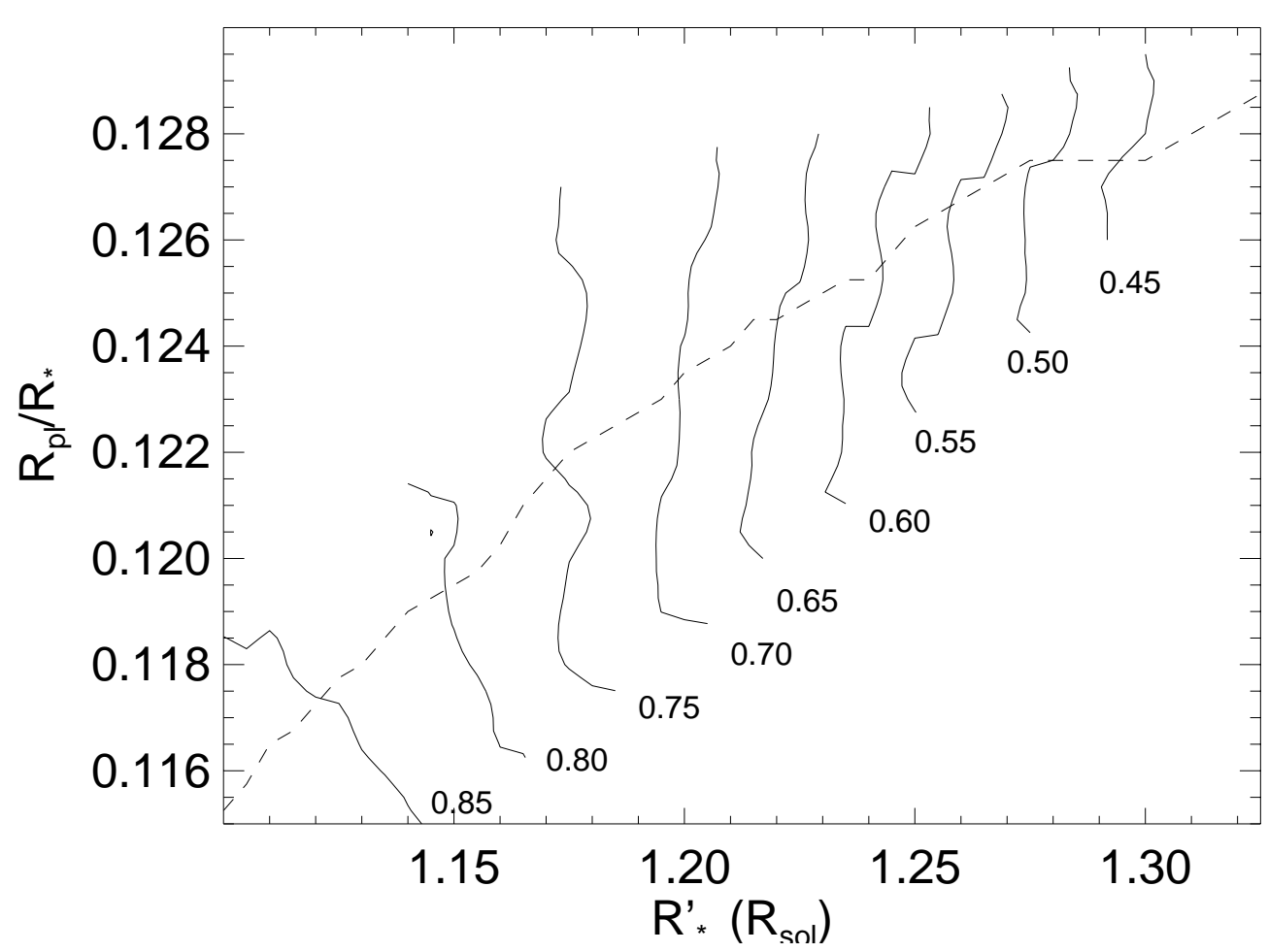

Fig. 3. The contours give limb darkening values ' $w^{\prime}$ for the combined $v+b$ color, as a function of the boundary parameters $R_{\mathrm{pl}} / R_{*}$ and $R_{*}$, for a fixed stellar mass of $1.1 M_{\odot}$ (or $R_{\mathrm{pl}} / R_{*}$ and $R_{*}^{\prime}$ for any stellar mass, see Sect. 3.2). The limited vertical lengths of the contours is given by the range in which solutions where calculated. The dashed line gives the trace of the best fit for each value of $R_{*}^{\prime}$ (see also Fig. 4). Small changes in the planet size (up-down) around the best fit have little effect on $w$.

input parameters following a Levenberg-Marquardt fitting algorithm. UFIT then stepped through a 3 dimensional grid of boundary values for $M_{*}, R_{*}$ and $R_{\mathrm{pl}} / R_{*}$, performing fits of the only free parameter $w$ at each step. The boundary values were varied within the following limits:

$0.95 M_{\odot} \leq M_{*} \leq 1.35 M_{\odot}$, with increments of $0.025 M_{\odot}$

$0.95 R_{\odot} \leq R_{*} \leq 1.35 R_{\odot}$, with increments of $0.025 R_{\odot}$

$0.11 \leq R_{\mathrm{pl}} / R_{*} \leq 0.145$, with increments of 0.00125

At each step, a new value for $\cos b$ was calculated from Eq.(2). If a physically meaningful solution for $\cos b$ was found, an initial value for $w$ was calculated from Eq.(4). Only if this value was in the range of $0.2 \leq w_{\text {init }}<1$, an actual fit to the light curve was performed. Since $w$ does not appear in Eq.(1), results from the fits will always comply with the boundary condition of the total transit duration $T_{14}$.

the representation of systems with several stars and planets, moons or rings. UFIT can perform fits to any of the input parameters of UTM. 


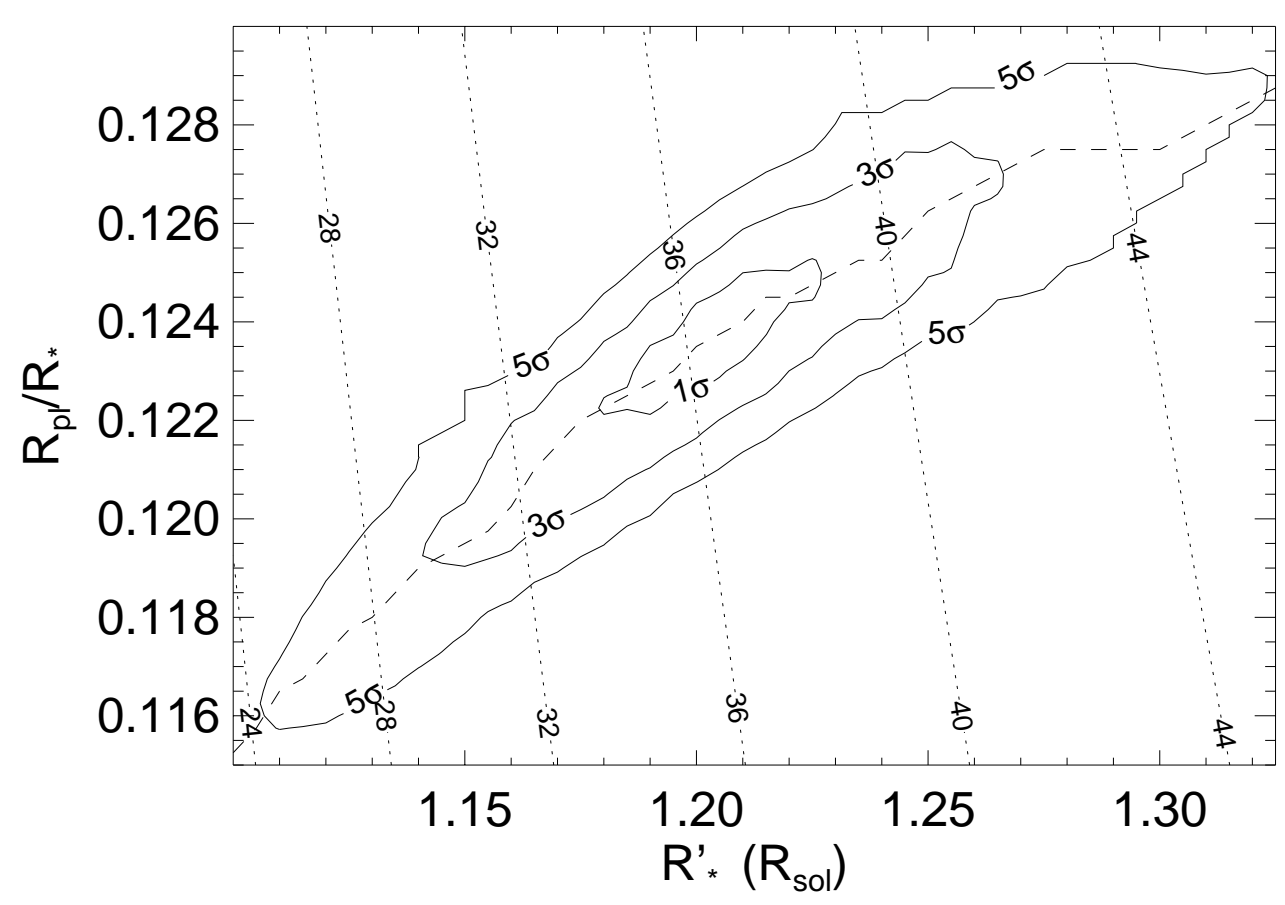

Fig. 4. Confidence regions $(1,3$ and $5 \sigma)$ of the best fit to $w$, within the same parameter space as the previous figure. The dashed line traces the best fitting value of $R_{\mathrm{pl}} / R_{*}$ for a given $R_{*}^{\prime}$. Dotted lines indicate the latitude (in degrees) of the transit, from Eq.(2).

The result from these 'scanning fits' are two three-dimensional arrays with the axes along $M_{*}, R_{*}$ and $R_{\mathrm{pl}} / R_{*}$, one containing values for the fitted limbdarkening coefficient $w$ and the other one the variances between fit and data. A 'cut' through such an array of $w$ values, from fits to the combined $v+b$ light curve, is shown in Fig. 3, and the corresponding variances, converted to confidence regions, are shown in Fig. 4. All confidences in this paper are calculated for 129 data points and 4 free parameters.

\subsection{Dependence on stellar mass and radius}

In the following, we will use the light curve with the lowest noise -the combined one from $v$ and $b$ filters- for the derivation of all parameters, except for the limb-darkening coefficients, which are wavelength dependent. In Fig. 5, the 3-dimensional array that resulted from the scanning fit is flattened into 2 dimensions $\left(M_{*}, R_{*}\right)$ by selecting those values of $R_{\mathrm{pl}} / R_{*}$ which gave the best fit at each point $M_{*}, R_{*}$. The best solution appears in a narrow band, which can be approximated by

$$
R_{*}=0.34 M_{*}+0.825( \pm 0.06),
$$




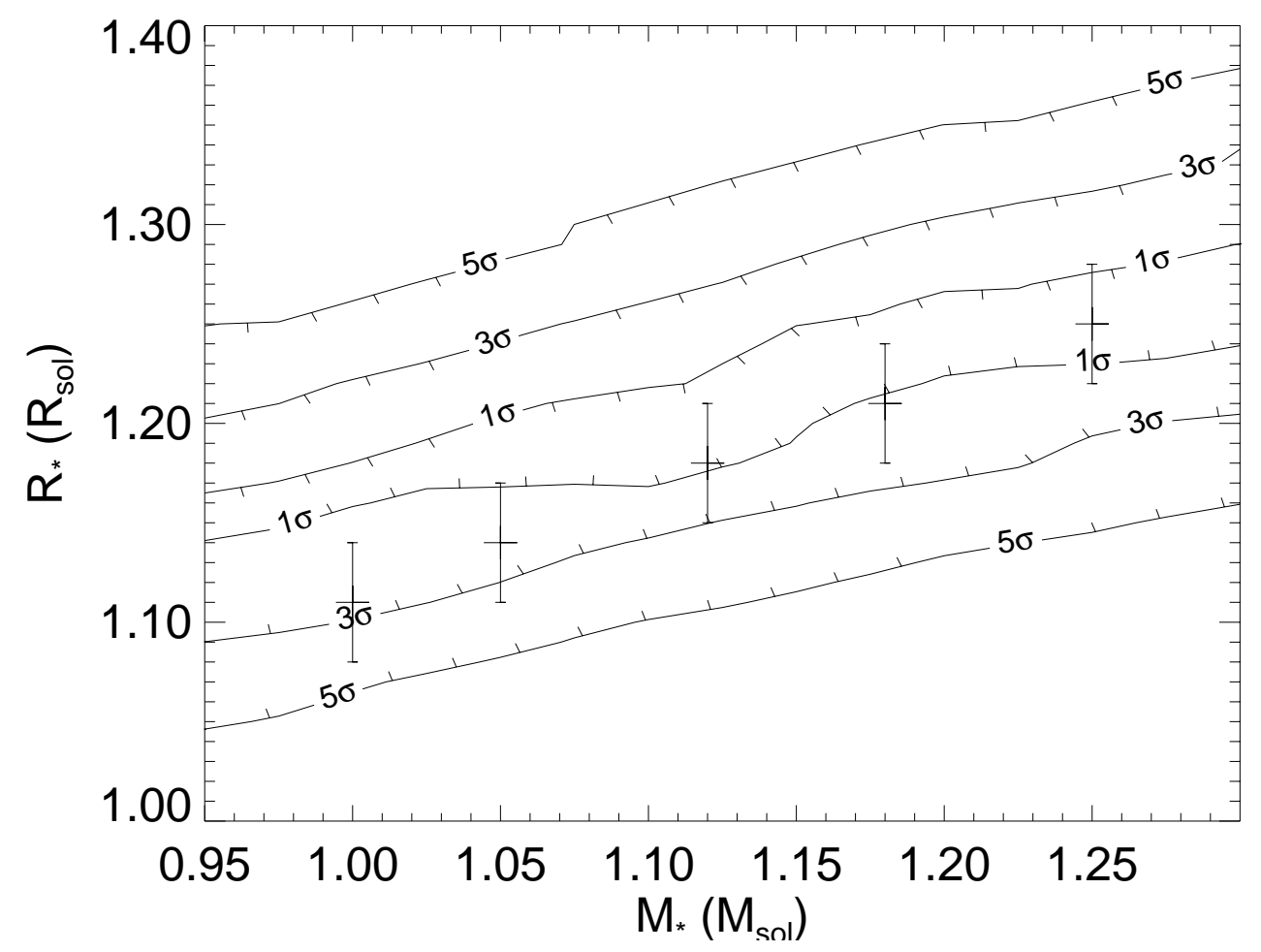

Fig. 5. 1, 3, and $5 \sigma$ confidence regions of the best fit on the combined $v$ and $b$ light curve, as a function of the mass and radius of the central star. Best fits lie along a band in $\left(M_{*}, R_{*}\right)$, also given by Eq.(5). The 5 points with error bars are those radii where limb-darkening coefficients from transit data and from ATLAS atmosphere models coincide (see Section 4 and Fig. 8).

with the errors indicating a $3 \sigma$ confidence limit. This relationship follows lines of constant transit latitude, which is dominated by $\cos b \propto v_{\text {orb }} / R_{*} \propto M_{*}^{1 / 3} / R_{*}$ for $R_{\mathrm{pl}}<<R_{*}$ (see Eq.2). It should be noted that this relationship has no physical meaning in the sense of compatibility with stellar models. It is simply a result from the transit light curve and the $T_{14}$ boundary condition, giving the range of possible radii as a function of the stellar mass that are compatible with transit observations. Eq.(5) allows us to express most results given in the further sections as functions of a 'normalized radius' $R_{*}^{\prime}$, which is related to the actual stellar mass and radius (currently known with large errors only) as follows:

$$
R_{*}^{\prime}=R_{*}+0.34\left(1.1-M_{*}\right)
$$

For $M_{*}=1.1$, normalized and actual radii are equal. It should be noted that the stellar parameters given by Mazeh et al. $\left(M_{*}=1.1 M_{\odot}, R_{*}=1.2 R_{\odot}\right)$ lie well within the $1 \sigma$ confidence region of Fig. 5 . The radius of $1.27 \pm 0.05 R_{\odot}$ from transit observations by Jha et al. (2000) is slightly outside, and the value of $1.146 \pm 0.050 R_{\odot}$ from HST observations (Brown et al.) is slightly inside our $3 \sigma$ confidence region (both times assuming a stellar mass of $1.1 M_{\odot}$ ), with error 
Table 2

Determinations of planet and star sizes in the HD 209458 system

\begin{tabular}{lccc}
\hline Reference & $R_{\mathrm{pl}}\left(R_{\mathrm{Jup}}\right)$ & $R_{*}\left(R_{\odot}\right)$ & $R_{\mathrm{pl}} / R_{*}$ \\
\hline Charbonneau et al., 2000 & $1.27 \pm 0.02$ & 1.10 & $0.1193 \pm 0.002$ \\
Henry et al., 2000 & $1.42 \pm 0.10$ & 1.15 & $0.1276 \pm 0.009$ \\
Mazeh et al., 2000 & $1.40 \pm 0.17$ & 1.20 & $0.1205 \pm 0.015$ \\
Jha et al., 2000 & $1.55 \pm 0.10$ & 1.27 & $0.1261 \pm 0.008$ \\
Brown et al., 2001 & $1.347 \pm 0.06$ & 1.146 & $0.1214 \pm 0.002$ \\
this work & $1.435 \pm 0.05$ & 1.20 & $0.1235 \pm 0.004$ \\
\hline
\end{tabular}

bars from the HST observations well overlapping with our determination.

\subsection{Planet size, transit latitude and inclination}

In Fig. 4, the confidence regions along $R_{\mathrm{pl}} / R_{*}$ and $R_{*}^{\prime}$ are shown. It should be noted, that the relative planet size $R_{\mathrm{pl}} / R_{*}$ is a function of $R_{*}^{\prime}$, whereas the absolute size $R_{\mathrm{pl}}$ is not. The region of fits with any significant meaning $\left(\sigma<3 \sigma_{\min }\right)$ is limited to a range of stellar and planetary radii given by: $R_{*}^{\prime}=1.20 \pm 0.06 R_{\odot}$ and $R_{\mathrm{pl}} / R_{*}=0.1235 \pm 0.004$. For a stellar radius of $1.2 R_{\odot}$, the derived planet size would then be $1.435 \pm 0.05 R_{\text {Jup }}$. There is considerable scatter in the literature among derivations for the absolute size of the planet (Table 2), which is mainly caused by differences in the assumed stellar size. The agreement is much better if relative planet sizes are considered, whose average from all listed publications is $R_{\mathrm{pl}} / R_{*}=0.1231 \pm 0.003$, which is very close to the value derived in this work.

The best fitting planet sizes in each of the four Strömgren colors are shown in Fig. 6. Their scatter around the mean is better than $\pm 3 \%$. This shows that the depths of the transit across the different colors agree with each other, and that the magnitude zero points in the light curves were correctly set. The remaining differences among the individual light curves will then primarily be caused by the dependence of limb-darkening on the wavelength.

Independently of the stellar mass, the range of possible transit latitudes is restricted to $b=36.2 \pm 6^{\circ}$ (Fig. 4, within the $3 \sigma$ confidence region). For $M_{*}=1.1 M_{\odot}$, this corresponds to an inclination of the planet's orbit of $i=$ $85.96 \pm 0.55^{\circ}$, given by:

$$
\cos i=\frac{R_{*}}{a_{\mathrm{pl}}} \sin b
$$




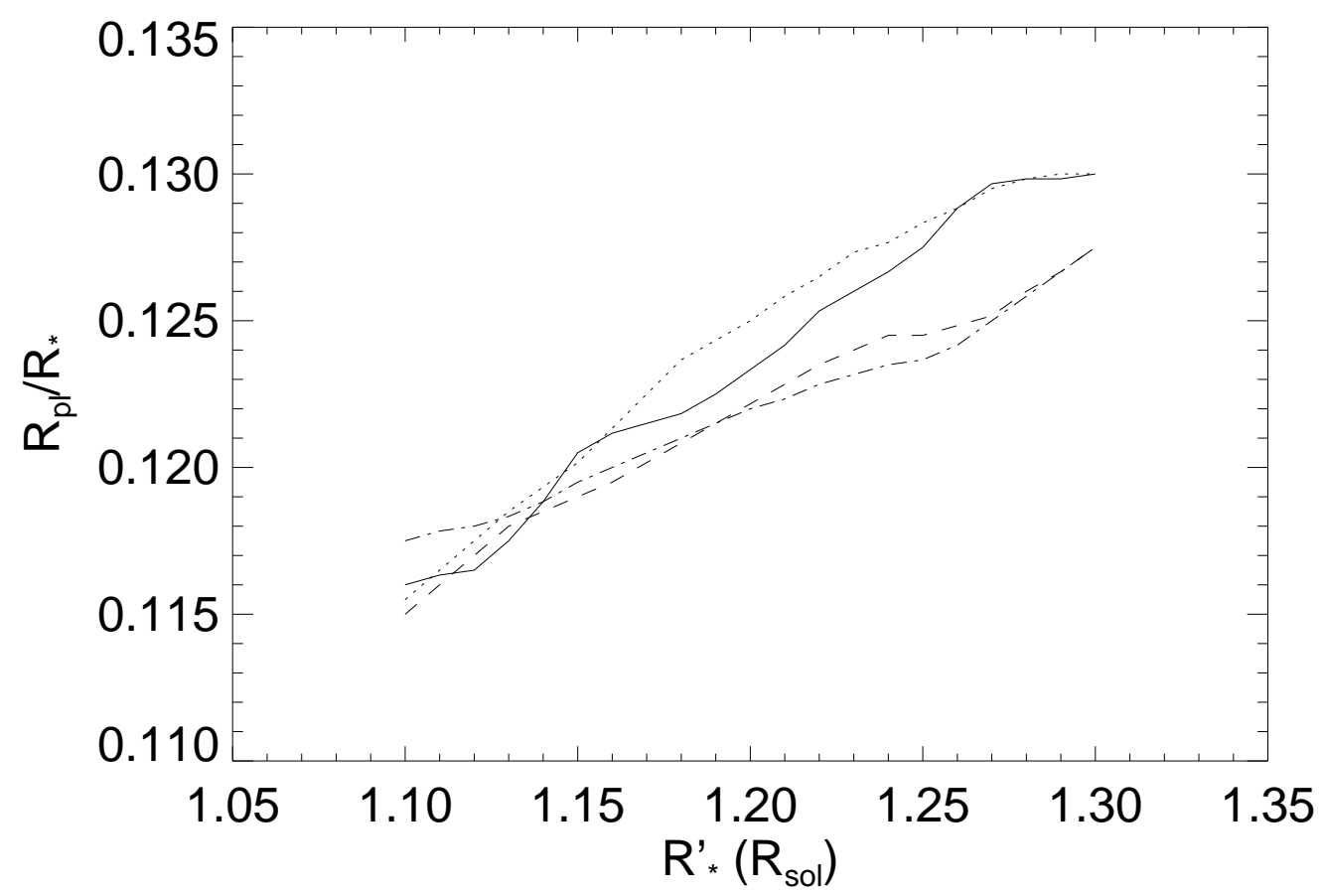

Fig. 6. Best fitting planet sizes in the four Strömgren colors, as a function of the stellar size ( $u$ : solid line; $v$ : dotted; $b$ : dashed; $y$ : dot-dashed)

where the planetary orbit's half axis $a_{\mathrm{pl}}\left(M_{*}, M_{\mathrm{pl}}, P\right)$ is given by Kepler's Third Law $\left(a_{\mathrm{pl}}=10.057 R_{\odot}\right.$ for $\left.M_{*}=1.1 M_{\odot}\right)$.

\section{The limb-darkening of HD 209458}

The excellent fit between the modeled light curves and data (Fig. 1) shows that the use of a linear limb-darkening expression has been adequate for this star.

Values of the limb-darkening are shown for the four Strömgren colors in Fig. 7, again quoting $w$ for the best fit at each value of $R_{*}^{\prime}$, similar to the dotted line in Fig. 3. Fig. 3 also shows that the derived values of $w$ are not very dependent on small deviations of $R_{\mathrm{pl}}$ around its best value. Individual errors of the limbdarkening coefficients are estimated at \pm 0.05 for $w_{v}$ and $w_{b}, \pm 0.07$ for $w_{y}$ and \pm 0.1 for $w_{u}$. The derived values agree reasonably well with the usual sequence of limb-darkening coefficients along the four colors, with a distance between $w_{u}$ and $w_{v}$ that is smaller than their uncertainty. It is also apparent, that only for a small range of stellar radii, reasonable limb-darkening values can be assigned to HD 209458. For two narrow wavelength ranges, centered around 590nm and $618 \mathrm{~nm}$, Brown et al. (2001) provide quadratic limb-darkening values, which 


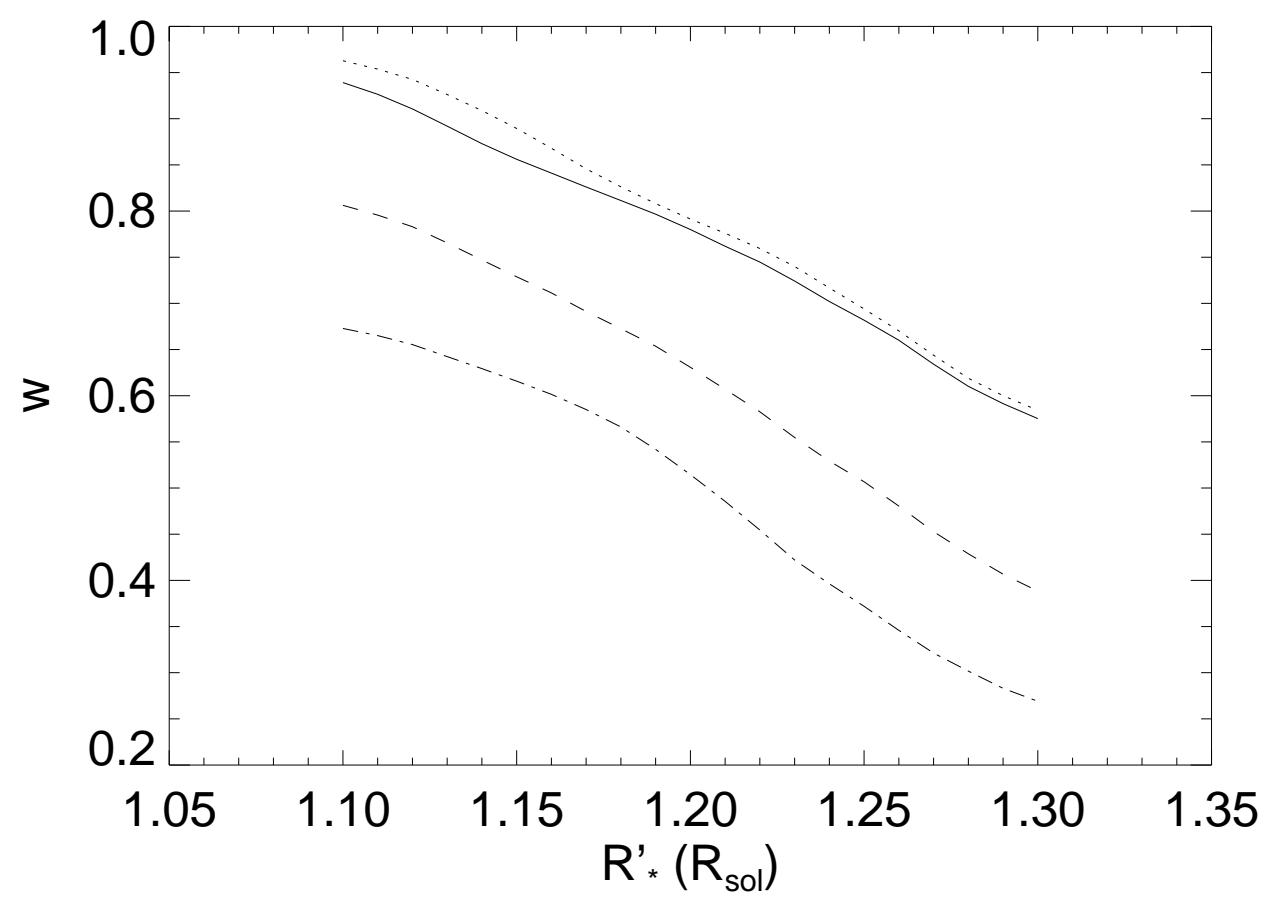

Fig. 7. Linear limb-darkening coefficients for HD 209458 in the Strömgren colors ( $u$ : solid line; $v$ : dotted; $b$ : dashed; $y$ : dot-dashed) as derived from the fits. They are plotted against the normalized stellar radius of Eq.(6) and correspond to traces along best fitting values of $R_{\mathrm{pl}} / R_{*}$, similar to the dashed line in Fig. 3.

correspond to linear coefficients of $w_{590} \approx 0.62$ and $w_{618} \approx 0.58$. These wavelength ranges are slightly red-wards of the Strömgren $y$ filter $(\lambda=547 \mathrm{~nm})$, and their limb-darkening agrees very well $w_{y} \approx 0.62$ from our data, assuming a stellar radius of $1.146 R_{\odot}$ as did Brown et al..

The limb-darkening coefficients have been compared with values derived from the combination of evolutionary stellar models (Claret, 1995) and stellar atmosphere calculations based on the ATLAS code, using tabulations of $w$ from Claret (1998a). All the models have solar abundances and surface gravity values were converted directly to stellar radii. Comparison with limb-darkening coefficients derived from PHOENIX code (Claret, 1998b) was not possible because the code does not cover the temperature range of HD 209458. The results from the evolutionary ATLAS models are shown in Fig. 8. Although a good general agreement between the limb-darkening sequence from transits and from ATLAS is found, the values from the transit have a wider spread across the four Strömgren colors. This can be seen through the intersections among the curves of $w$, which do not correspond to exactly the same stellar radii across the four colors. However, the intersections appear close to those stellar radii at which the best fits have been obtained. These intersection radii, averaged among the colors, are displayed in Fig. 5(crosses) and have 


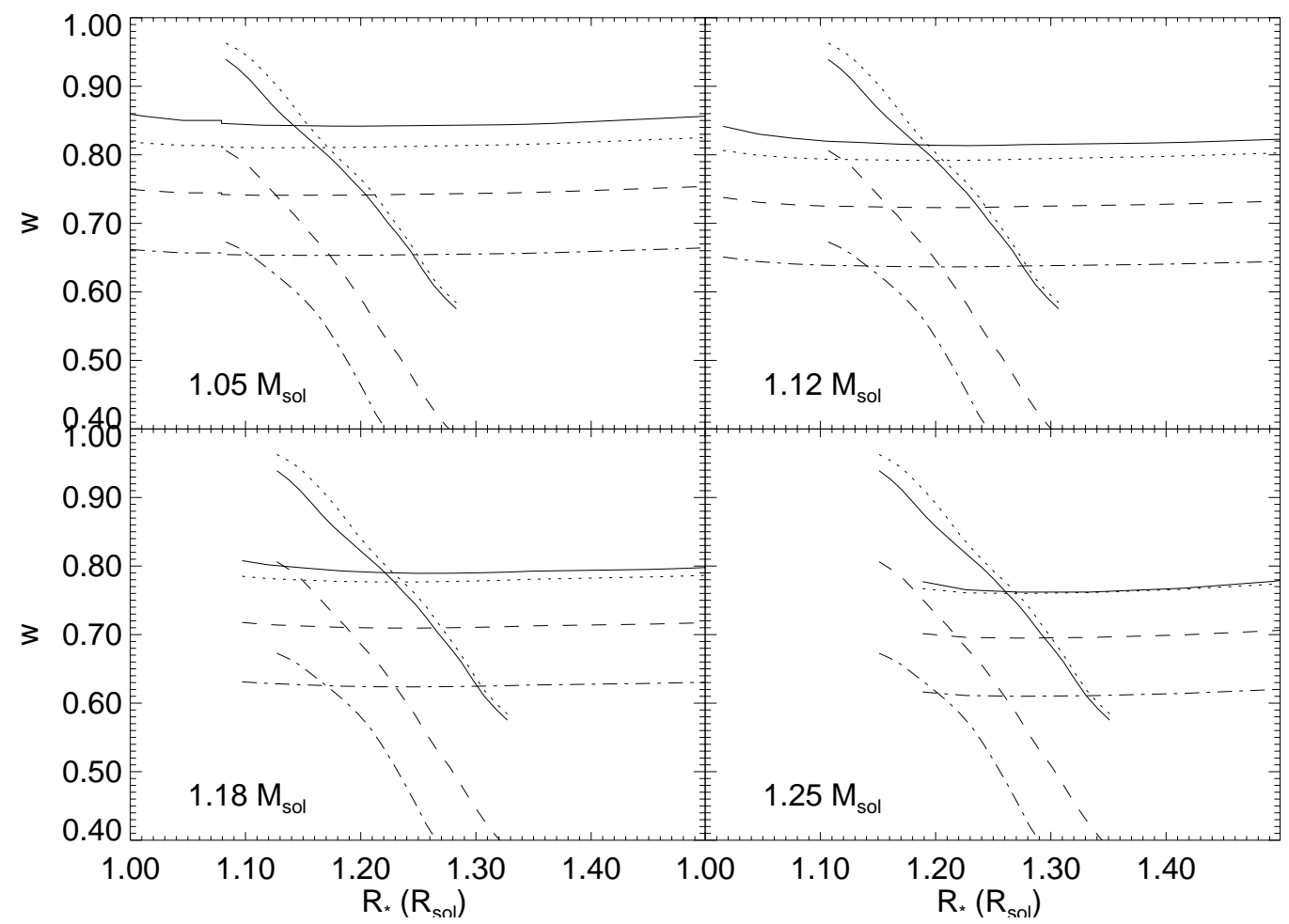

Fig. 8. Limb darkening coefficients derived from the transit data (inclined curves) against values based on the ATLAS model atmosphere combined with evolutionary stellar models (Claret, 1998a, flat curves). Plots are shown for stellar masses of 1.05 - $1.25 M_{\odot}$, with the normalized radii $\left(R_{*}^{\prime}\right)$ of Fig. 7 converted to $R_{*}$ using Eq.(6). Strömgren filters correspond to line-styles as in the previous figures.

a somewhat steeper dependency on $M_{*}$ than the radii directly derived from transits. For $M_{*}>1.25 M_{\odot}$, no stellar model delivers radii small enough for those derived from transits, and for masses less than $1.05 M_{\odot}$, radii from ATLAS correspond to transit-radii outside of the $2 \sigma$ confidence region. The radii based on limb-darkening provide thereby a constraint for the stellar mass to be within 1.05 and $1.25 M_{\odot}$, with a stellar mass of $1.1 M_{\odot}$ leading to a radius of $1.17 \pm 0.03 R_{\odot}$.

The standard linear limb-darkening expression appears to be sufficient to account for our observations. For one, HD 209458 is in the temperature range where the linear expression is expected to give good results. Second, most of the contributing non-linearity would come from angles close to the stellar rim, which are within the ingress and egress zone of the transit. Parameters describing non-linearities are therefore susceptible to introduce ambiguities with other transit parameters. Lastly, the number of free parameters in fits should be kept small in order to avoid the derivation of highly interdependent parameters of low significance. For stars outside of the temperature range of 5000-6000 K, single-parametric limb-darkening expressions different to the one used here may be devised from modeled surface brightness distributions. An 
Table 3

Derived parameters for the HD 209458 system, and their interdependencies

\begin{tabular}{lccc}
\hline Parameter & Value & Assumption & Reference \\
\hline$R_{*}$ & $1.20 \pm 0.02 R_{\odot}$ & $M_{*}=1.1 M_{\odot}$ & Fig. 5, Eq. (5) \\
$R_{\mathrm{pl}} / R_{*}$ & $0.1235 \pm 0.001$ & $R_{*}^{\prime}=1.20 \pm 0.02 R_{\odot}$ & Figs. 4,6 \\
$R_{\mathrm{pl}} / R_{\text {Jup }}$ & $1.435 \pm 0.05$ & $M_{*}=1.1 M_{\odot}, R_{*}=1.20 \pm 0.02 R_{\odot}$ & Figs. 4,6 \\
$b$ & $36.2 \pm 2.2^{\circ}$ & $R_{\mathrm{pl}} / R_{*}$ and $R_{*}^{\prime}$ from above & Eq. (2), Fig. 4 \\
$i$ & $85.96 \pm 0.22^{\circ}$ & $b$ and $M_{*}$ from above & Eq. $(7)$ \\
$w_{u}$ & $0.82 \pm 0.10$ & $R_{*}^{\prime}=1.17 R_{\odot}$ & Fig. 7 \\
$w_{v}$ & $0.84 \pm 0.05$ & " & " \\
$w_{b}$ & $0.69 \pm 0.05$ & " & " \\
$w_{y}$ & $0.59 \pm 0.07$ & & \\
\hline
\end{tabular}

example that this can be done with a very high precision, describing limbdarkening of the Sun, is given by Hestroffer and Magnan (1998).

A possible planetary atmosphere may also cause differential color effects to become apparent in transit light curves. A color dependency of the atmospheric transparency, albedo, or scale height will cause the planet to display an effective radius that changes with colors. We however do not expect to detect such color effects within the precision of our data. Assuming a precision of $0.5 \mathrm{mmag}$, then $\delta L \approx \delta A_{\mathrm{pl}} / A_{*}$, where $A_{*}=\pi R_{*}^{2}$ is the cross section of the star, and $\delta A_{\mathrm{pl}}$ is the differential cross section of the planet. With $\delta A_{\mathrm{pl}}$ depending on a variation of the planetary radius like: $\delta A_{\mathrm{pl}}=2 \pi R_{\mathrm{pl}} \delta R_{\mathrm{pl}}$, we may solve for $\delta R_{\mathrm{pl}}$ as follows: $\delta R_{\mathrm{pl}}=\delta A_{\mathrm{pl}} / 2 \pi R_{\mathrm{pl}}=R_{*}^{2} \delta L / 2 R_{\mathrm{pl}}$. For $R_{\mathrm{pl}}=1.43 R_{\mathrm{Jup}}=$ $0.148 R_{\odot}$ and $R_{*}=1.20 R_{\odot}$ we obtain $\delta R_{\mathrm{pl}}=0.0024 R_{\odot} \approx 1700 \mathrm{~km}$, meaning that color variations induced in the effective radius need to be larger than that value to be detectable in our data. Unless HD 209458 has an extremely extended atmosphere with a very unlikely transparency dependence along the Strömgren colors, our data will not contain any signatures from the planetary atmosphere. Data with significantly higher precision may however very well indicate the presence of planetary atmospheres.

\section{Conclusions}

A major goal of this work has been the derivation of limb-darkening coefficients for the star HD 209458. These, and other parameters of the system, have been obtained in a way which is quite independent of the underlying physical 
assumptions. An overview of the derived parameters and assumptions is given in Table 3. References in the table are given to understand the interdependence of the derived parameters upon the assumed values. Any improvement in any parameter could be easily used, through the references given in the table, to adjust any other parameter for the HD 209458 system. For example, if we assume the stellar size of $1.146 R_{\odot}$ from HST observations by Brown et al., and a mass of $1.1 M_{\odot}$, the corresponding planet size, transit latitude, and orbital inclination derived from our data would be $R_{\mathrm{pl}}=1.323 R_{\mathrm{Jup}}, b=30.0^{\circ}$ and $i=86.73^{\circ}$, which is in very good agreement with values cited by these authors.

The derived limb-darkening coefficients are in good agreement with the ATLAS atmospheric models, combined with evolutionary stellar models, over a stellar masses range within 1.05 to $1.25 M_{\odot}$. There is a minor difference between the stellar radii which best fit the transit light curve $\left(1.20 \pm 0.02 R_{\odot}\right.$ for $\left.M_{*}=1.1 M_{\odot}\right)$, and the radii which produce the limb-darkenings best describing the ATLAS models $\left(1.17 \pm 0.03 R_{\odot}\right)$. As the differences are within the error bars, we can not distinguish if they are caused by the errors inherent in the observational data, or by the calibration of the ATLAS models. More transit observations of HD 209458 with the same instrument are planned for the next year. It will then be possible to co-add light curves from individual transits, giving rise to an improvement in the precision of the parameters derived in this paper, especially with regard to the limb-darkening.

With the discovery of further transiting systems, and with the availability of very high precision data from space missions, we expect that transit observation will become an important tool in the verification of model atmospheres, and will be able to constrain stellar structure models over a wide range of stellar types.

\section{Acknowledgments}

We thank D. Charbonneau and T. Brown to forward us some of the parameters they derived from observations with the Hubble Space Telescope, and previous to publication. We thank the anonymous referee for his comments, and both him and the Editor for their rapid responses. The Observatorio de Sierra Nevada (OSN) is operated by the Instituto de Astrofísica de Andalucía.

\section{References}

T. Brown, D. Charbonneau, R. L. Gilliland, R. W. Noyes, and A. Burrows. tbd. ApJL, submitted, 2001. 
T. Castellano, J. Jenkins, D. E. Trilling, L. Doyle, and D. Koch. Detection of planetary transits of the star HD 209458 in the Hipparcos data set. ApJL, 532:L51-L53, Mar. 2000.

D. Charbonneau, T. M. Brown, D. W. Latham, and M. Mayor. Detection of planetary transits across a sun-like star. ApJL, 529:L45-L48, Jan. 2000.

A. Claret. Stellar models for a wide range of initial chemical compositions until helium burning. I. From $\mathrm{x}=0.60$ to $\mathrm{x}=0.80$ for $\mathrm{z}=0.02$. $A \& A S, 109$ : 441-446, Mar. 1995.

A. Claret. Comprehensive tables for the interpretation and modeling of the light curves of eclipsing binaries. A\&SAS, 131:395-400, Sept. 1998a.

A. Claret. Very low mass stars: non-linearity of the limb-darkening laws. $A \mathscr{E} A$, 335:647-653, July 1998b.

B. Gronbech, E. H. Olsen, and B. Stromgren. Standard stars uvby photoelectric photometry south of declination +10 . A\&AS, 26:155-176, Oct. 1976.

G. W. Henry, G. W. Marcy, R. P. Butler, and S. S. Vogt. A transiting "51 Peg-like" planet. ApJL, 529:L41-L44, Jan. 2000.

D. Hestroffer and C. Magnan. Wavelength dependency of the solar limb darkening. A $\mathcal{E} A, 333: 338-342$, May 1998.

S. Jha, D. Charbonneau, P. M. Garnavich, D. J. Sullivan, T. Sullivan, T. M. Brown, and J. L. Tonry. Multicolor observations of a planetary transit of HD 209458. ApJL, 540:L45-L48, Sept. 2000.

T. Mazeh, D. Naef, G. Torres, D. W. Latham, M. Mayor, J. Beuzit, T. M. Brown, L. Buchhave, M. Burnet, B. W. Carney, D. Charbonneau, G. A. Drukier, J. B. Laird, F. Pepe, C. Perrier, D. Queloz, N. C. Santos, J. Sivan, S. . Udry, and S. Zucker. The spectroscopic orbit of the planetary companion transiting HD 209458. ApJL, 532:L55-L58, Mar. 2000.

N. Robichon and F. Arenou. HD 209458 planetary transits from Hipparcos photometry. A\&BA, 355:295-298, Mar. 2000.

S. Soderhjelm. Possible detection of the planet transits of HD 209458 in the Hipparcos photometry. Informational Bulletin on Variable Stars, 4816:1+, Dec. 1999. 